\title{
Multivariable Iterative Learning Control Design Procedures: From Decentralized to Centralized, Illustrated on an Industrial Printer
}

\author{
Lennart Blanken, Tom Oomen
}

\begin{abstract}
Iterative Learning Control (ILC) enables high control performance through learning from measured data, using only limited model knowledge in the form of a nominal parametric model to guarantee convergence. The aim of this paper is to develop a range of approaches for multivariable ILC, where specific attention is given to addressing interaction. The proposed methods either address the interaction in the nominal model, or as uncertainty, i.e., through robust stability. The result is a range of techniques, including the use of the structured singular value (SSV) and Gershgorin bounds, that provide a different trade-off between modeling requirements, i.e., modeling effort and cost, and achievable performance. This allows an appropriate choice in view of modeling budget and performance requirements. The trade-off is demonstrated in a case study on an industrial printer.
\end{abstract}

\section{INTRODUCTION}

Iterative Learning Control (ILC) can significantly improve the control performance of systems that perform repeating tasks. After each repetition, or trial, the control action is improved by learning from past trials using an approximate model of the system. Many successful applications have been reported, including additive manufacturing [1], microscopic imaging [2], printing systems [3], and wafer stages [4].

The observation that many ILC applications are inherently multivariable has lead to developments of ILC theory for multivariable systems. Most design algorithms for multivariable ILC have been developed in the so-called lifted or supervector framework [5], where the ILC controller follows from a normoptimization problem over a finite-time horizon, see, e.g., [6].

Robust convergence properties of ILC algorithms, i.e., robust stability in trial-domain, are crucial to deal with modeling errors. Optimization-based algorithms have been further extended to address robust stability in, e.g., [4], [7], [8], [9], [10]. These approaches rely on a detailed specification of the nominal model and its uncertainty in a certain prespecified form. Despite being very systematic, this imposes a large burden on the model requirements, since modeling of uncertainty often requires substantial effort of the user [11]. Alternatively, fully data-driven ILC algorithms have been developed in, e.g., [12], but these require a high experimental cost.

Although robust multivariable ILC has been significantly developed, especially from a theoretical perspective, these approaches are often not employed due to high requirements

The authors are with the Department of Mechanical Engineering, Eindhoven University of Technology, 5600 MB Eindhoven, The Netherlands (e-mail: 1.1.g.blanken@tue.nl; t.a.e.oomen@tue.nl). This work is supported in part by Océ Technologies, and in part by the Netherlands Organisation for Scientific Research (NWO) through research programme VIDI under project 15698. on uncertainty modeling. The aim of the present paper is to develop a range of user-friendly multivariable ILC design approaches. Indeed, in many applications, ILC controllers are designed in the frequency-domain [5]. Compared to the normoptimal framework, this enables a systematic and inexpensive robust design in the sense of modeling requirements, especially regarding the uncertainty. Accurate and inexpensive frequency response function (FRF) measurements [13] can be employed to model the uncertainty, see [4], [14]. In addition, frequencydomain design allows for manual loop-shaping, which is often preferred by control engineers. However, since such design approaches are mainly single-input single-output (SISO), design for multiple-input multiple-output (MIMO) systems typically involves their application to multiple SISO loops, see, e.g., [5], [15]. Interaction is typically ignored, which can lead to stability issues, i.e., non-converging algorithms. This is especially crucial for ILC, since its control action is effective up to the Nyquist frequency [16].

The seemingly drastical increase in required modeling effort to enforce robust convergence of multivariable ILC algorithms must be justified by the imposed performance requirements. Interestingly, interaction is typically addressed through full MIMO, or centralized, ILC design. Successful MIMO design approaches include $\mathcal{H}_{\infty}$ synthesis [4], [17], the more restricted class of P-type ILC [18], and gradient-based algorithms for point-to-point tracking [19]. Centralized techniques enable robust convergence and superior performance, yet require a MIMO parametric model of the system, including interaction. These models can be difficult and expensive to obtain, especially for lightly damped mechatronic systems due to complex dynamics and numerical issues [20].

The main contribution of this paper is a systematic design framework for analysis and synthesis of multivariable ILC, that explicitly addresses the design trade-offs between modeling and performance requirements. The proposed solutions, which form subcontributions, range from decentralized to centralized designs, with various levels of modeling requirements. The decentralized designs build on results in, e.g., [21] to guarantee robust convergence, including the use of the structured singular value [22, Chapter 11], and require limited user effort using only SISO parametric models. The effectiveness of the framework is demonstrated in a case study on an industrial flatbed printer. The paper extends preliminary results in [23], [24] through the design framework, new technical results, detailed proofs, and application results.

Notation. The imaginary unit is denoted $\iota$, i.e., $\iota^{2}=-1$. 


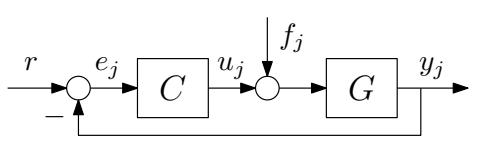

Fig. 1. ILC control configuration.

\section{Problem Formulation}

\section{A. ILC Setup}

Consider the control configuration in Figure 1, consisting of possibly non-square plant $G \in \mathcal{R}^{n_{y} \times n_{u}}(z)$ and internally stabilizing feedback controller $C \in \mathcal{R}^{n_{u} \times n_{y}}(z)$. The disturbance $r \in \ell_{2}$ is trial-invariant, where each repetition, or trial, is denoted by index $j \in \mathbb{N}_{\geq 0}$. The aim is to minimize the tracking error $e$ in the presence of $r$. Note that trial-varying disturbances are tacitly omitted, see, e.g., [25], [26] for details. The output in trial $j$ is denoted $y_{j}$, the feedforward by $f_{j}$, and

$$
e_{j}=S r-J f_{j},
$$

with sensitivity function $S=(I+G C)^{-1} \in \mathcal{R H}_{\infty}^{n_{y} \times n_{y}}$, and process sensitivity function $J=S G \in \mathcal{R} \mathcal{H}_{\infty}^{n_{y} \times n_{u}}$. Zero initial conditions are assumed without loss of generality [5]. If $G$ is stable, then $C=0$ is admissible such that $S=I$ and $J=G$.

The objective of ILC is to improve control performance in the next trial $j+1$ by selecting the command input $f_{j+1}$. Typically, an algorithm of the following form is invoked:

$$
f_{j+1}=Q\left(f_{j}+L e_{j}\right),
$$

where $L \in \mathcal{R} \mathcal{L}_{\infty}^{n_{u} \times n_{y}}$ and $Q \in \mathcal{R} \mathcal{L}_{\infty}^{n_{u} \times n_{u}}$ are to be designed. Notice $L, Q$ can be non-causal, since (2) is computed off-line.

\section{B. ILC Design for SISO Systems}

For the case $n_{u}=n_{y}=1$, design procedures are well developed. Often a two-step approach is used, see, e.g., [14].

\section{Procedure 1. Frequency-domain SISO ILC design}

1) Choose $L(z)$ such that $L\left(e^{\iota \omega}\right) J\left(e^{\iota \omega}\right) \approx 1, \omega \in\left[0, \omega_{c}\right]$. This step requires a parametric model of $J(z)$.

2) For robust stability, $Q(z)$ is selected as a low-pass filter with cut-off frequency near $\omega_{c}$, such that $Q\left(e^{\iota \omega}\right) \approx 0, \forall \omega>\omega_{c}$. This can be performed using nonparametric models of $J\left(e^{\iota \omega}\right)$.

Procedure 1 requires limited model knowledge, since robust stability can be guaranteed through FRFs, see, e.g., [13], which are for mechatronic systems often accurate and fast to obtain.

A naive extension of Procedure 1 to the multivariable case could be to implement multiple SISO ILC. In this paper, it is demonstrated that this can lead to non-convergent algorithms.

\section{Problem Formulation and Contributions}

The problem considered in this paper is the design of multivariable filters $L(z)$ and $Q(z)$ in (2) in the frequency domain with respect to the following requirements:

R1) Robust convergence of (2), i.e., stability in trial domain;

R2) High control performance, i.e., a small error $e_{j}$;

R3) Limited required user effort.

The term user effort relates to design tools and required models, i.e., parametric vs. nonparametric, and SISO vs. MIMO.

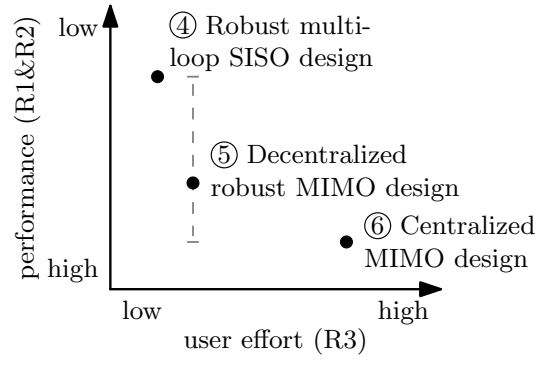

Fig. 2. Schematic overview of approaches in the design framework, illustrating the trade-offs between performance (R1 and R2), and user effort (R3). Depending on the level of interaction, the vertical position of (5) may vary.

The main contribution is the development of a step-by-step design procedure for multivariable iterative learning control that addresses modeling and robustness aspects. The proposed design techniques vary in sophistication, and range from

- decentralized designs, using SISO parametric models, to

- centralized designs, requiring MIMO parametric models, where in all cases, robustness to modeling errors is addressed using nonparametric FRF measurements. The procedure generalizes Procedure 1 to the MIMO case, and provides a coherent overview of available approaches, such that a well-motivated choice can be made for the problem at hand in view of R1-R3.

\section{Overview of Design Framework and Outline of Paper}

The present paper addresses theoretical, design, and algorithmic aspects to obtain a practically implementable design framework for MIMO ILC. The framework connects all design approaches, see Figure 2, and is summarized next.

\section{Procedure 2. Frequency-domain MIMO ILC design}

(1) Non-parametric modeling.

- Identify MIMO FRF model $\hat{J}_{\mathrm{FRF}}\left(e^{\iota \omega}\right)$ of $J$, see, e.g., [13].

(2) Interaction analysis. Decoupled?

- If yes: independent SISO design (Procedure 1).

(3) Decoupling transformations. Decoupled?

- If yes: independent SISO design (Procedure 1).

(4) Robust multi-loop SISO design (Section IV).

i) Obtain SISO parametric models $\widehat{J_{i i}}(z)$;

ii) Robust multi-loop SISO design of $L(z)=\operatorname{diag}\left\{L_{i i}(z)\right\}$ and $Q(z)$ using $\widehat{J_{i i}}(z)$ and FRF model $\hat{J}_{\mathrm{FRF}}\left(e^{\iota \omega}\right)$ (Algorithm 1).

Performance not satisfactory? Proceed to next step.

(5) Decentralized robust MIMO design (Section V).

i) Decentralized design of $L(z)$ and $Q(z)$ for robustness to deliberately ignored interaction and modeling errors, using $\widehat{J_{i i}}(z)$ and FRF model $\hat{J}_{\text {FRF }}\left(e^{\iota \omega}\right)$ (Algorithm 2).

Performance not satisfactory? Proceed to next step.

6) Centralized MIMO design (Section VI).

i) Obtain MIMO parametric model $\hat{J}(z)$, including interaction;

ii) MIMO design of $L(z)$ and $Q(z)$ for robustness to modeling errors, using $\hat{J}(z)$ and FRF model $\hat{J}_{\mathrm{FRF}}\left(e^{\iota \omega}\right)$ (Algorithm 3).

The key point is that modeling requirements should only be increased if justified by performance requirements. Indeed, (2) to (5) require only SISO parametric models and an FRF measurement, and may yield satisfactory performance, while 6) requires a costly MIMO parametric model. 


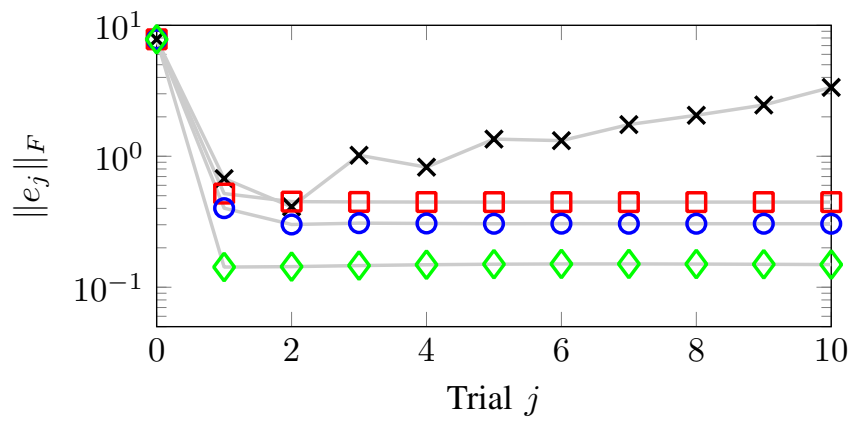

Fig. 3. Simulation results: often-used multi-loop SISO ILC design $(X)$ can lead to non-convergent algorithms. Through the developed design approaches in steps (4) ( $\square$ ), (5) (O), and (6) ( $\diamond)$, a well-motivated balance can be made between achievable performance, i.e., norm of the asymptotic error $\left\|e_{\infty}\right\|_{F}$, and the associated user effort in terms of modeling cost and design complexity.

Remark 1. Plant uncertainty can directly be addressed in Procedure 2 through confidence intervals of FRF estimates.

The outline of the paper is as follows. First, the design problem is analyzed. Then, in Sections IV to VI, the design techniques are developed that constitute steps (4) to (6). In Section VII, Procedure 2 is applied to a multivariable case study. A preview on the results is presented in Figure 3, illustrating the trade-offs between approaches in Procedure 2.

\section{AnAlysis of ILC Design Problem}

In this section, the general ILC algorithm (2) is analyzed, and robust convergence and control performance are defined.

\section{A. Convergence and Performance}

Combining (1) and (2) yields the linear iterative systems that describe the propagation of $e_{j}$ and $f_{j}$ in the trial domain:

$$
\begin{aligned}
& f_{j+1}=Q(I-L J) f_{j}+Q L S r \\
& e_{j+1}=J Q(I-L J) J_{l}^{-1} e_{j}+\left(I-J Q J_{l}^{-1}\right) S r .
\end{aligned}
$$

where (4) holds if a left inverse $J_{l}^{-1}$ exists such that $J_{l}^{-1} J=$ $I$, i.e., at least $n_{y} \geq n_{u}$. Convergence is formalized next.

Definition 1. System (3) is convergent iff for all $r, f_{0} \in \ell_{2}$, there exists an asymptotic signal $f_{\infty} \in \ell_{2}$ such that

$$
\limsup _{j \rightarrow \infty}\left\|f_{\infty}-f_{j}\right\|=0 .
$$

Then, the asymptotic signals $f_{\infty}$ and $e_{\infty}$ are obtained as

$$
\begin{aligned}
& f_{\infty}=\lim _{j \rightarrow \infty} f_{j}=(I-Q(I-L J))^{-1} Q L S r, \\
& e_{\infty}=\lim _{j \rightarrow \infty} e_{j}=\left(I-J(I-Q(I-L J))^{-1} Q L\right) S r .
\end{aligned}
$$

A condition for convergence of (3) and (4) is given next.

Theorem 1. Iterations (3), (4) converge to $f_{\infty}$ and $e_{\infty}$ iff

$$
\rho\left(Q\left(e^{\iota \omega}\right)\left(I-L\left(e^{\iota \omega}\right) J\left(e^{\iota \omega}\right)\right)<1 \quad \forall \omega \in[0, \pi],\right.
$$

where $\rho(\cdot)$ denotes spectral radius, i.e., $\rho(\cdot)=\max _{i}\left|\lambda_{i}(\cdot)\right|$.

See [27, Theorem 6] for a proof, which can be appropriately extended for non-causal $L, Q \in \mathcal{R} \mathcal{L}_{\infty}$. Although (6) guarantees convergence, it does not guarantee good learning transients. Monotonic convergence is considered next.

Definition 2. Iteration (3) converges monotonically w.r.t. the $\ell_{2}$ norm of $f_{j}$ to $f_{\infty}$ with convergence rate $\gamma, 0 \leq \gamma<1$, iff

$$
\left\|f_{\infty}-f_{j+1}\right\|_{\ell_{2}} \leq \gamma\left\|f_{\infty}-f_{j}\right\|_{\ell_{2}} \quad \forall j
$$

Theorem 2. Iteration (3) converges monotonically w.r.t. the $\ell_{2}$ norm of $f_{j}$ to fixed point $f_{\infty}$, with convergence rate $\gamma$, iff

$$
\gamma:=\|Q(I-L J)\|_{\infty}<1
$$

where $\|H(z)\|_{\infty}=\sup _{\omega \in[0, \pi]} \bar{\sigma}\left(H\left(e^{\iota \omega}\right)\right)$ is the $\mathcal{L}_{\infty}$-norm, and $\bar{\sigma}$ denotes the maximum singular value.

See [26, Theorem 2] for a proof. Note (7) is equivalent to

$$
\bar{\sigma}\left(Q\left(e^{\iota \omega}\right)\left(I-L\left(e^{\iota \omega}\right) J\left(e^{\iota \omega}\right)\right)\right)<1 \quad \forall \omega \in[0, \pi] .
$$

In view of (5), the following result is crucial for performance.

Theorem 3. Assume $L\left(e^{\imath \omega}\right), J\left(e^{\imath \omega}\right) \neq 0, \forall \omega$, in iteration (3). Given that (6) holds, then for all $r \in \ell_{2}, e_{\infty}=0$ iff $Q=I$.

Proof. Since (6) holds, the fixed points $e_{\infty}$ and $f_{\infty}$ exist.

$(\Leftarrow)$ If $Q=I$, then $f_{\infty}=f_{\infty}+L e_{\infty}$, which implies $e_{\infty}=0$. $(\Rightarrow)$ If $e_{\infty}=0$, then $f_{\infty}=Q f_{\infty}$, which implies $Q=I$.

\section{B. Design and Modeling Considerations}

Theorems 1, 2 and 3 have direct consequences for the design of $L$ and $Q$. To achieve $e_{\infty}=0, L$ should be designed such that $\rho(I-L J)<1$, and $Q$ must equal $I$. For fast convergence, $\|Q(I-L J)\|_{\infty}$ should be small.

\section{Multi-Loop SiSO ILC Design}

For systems where interaction is absent or sufficiently small, possibly after a decoupling process, multiple SISO ILC can be designed, see (2) and (3). In this section, it is shown that in the presence of interaction, multi-loop SISO designs may lead to non-convergent schemes, i.e., R1 is not guaranteed. To account for ignored interaction, the ILC can be robustified a posteriori, which is shown to lead to conservatism, hence compromising R2. It is assumed that $J$ is square, i.e., $n \equiv n_{u}=n_{y}$, possibly after a squaring-down process, see, e.g., [28].

\section{A. Independent SISO ILC Design for MIMO Systems}

If no coupling is present, i.e., $J(z)=\operatorname{diag}\left\{J_{i i}(z)\right\}$, then multi-loop SISO filters $L$ and $Q$ can be designed by application of Procedure 1 to each loop $i=1, \ldots, n$. That is, design

$$
L=\operatorname{diag}\left\{l_{1}, l_{2}, \ldots, l_{n}\right\}, \quad Q=\operatorname{diag}\left\{q_{1}, q_{2}, \ldots, q_{n}\right\},
$$

according to the set of SISO criteria

$$
\left|q_{i}\left(e^{\iota \omega}\right)\left(1-l_{i}\left(e^{\iota \omega}\right) J_{i i}\left(e^{\iota \omega}\right)\right)\right|<1 \quad \forall i, \omega \in[0, \pi] .
$$

Typically, each $l_{i}(z)$ is based on inversion of $\widehat{J_{i i}}(z)$, see, e.g., [5], [15], and [17], [29] for algorithms. Often, $q_{i}(z)$ are zero-phase filters, and are implemented non-causally, i.e., an operation with $q_{i}$ and its adjoint $q_{i}^{*}$, see, e.g., [12], [14].

However, when considerable interaction is present, independent SISO designs may lead to non-convergent systems, see also Figure 3. Indeed, (10) does not guarantee Theorem 1. 


\section{B. Accounting for Ignored Interaction Through Robustness}

Several approaches can be taken based on Theorems 1 and 2 to enable SISO design of $Q$ for robust MIMO convergence. Their restrictiveness is subject to a trade-off with the assumptions made on the structure of $Q$. Selecting $Q(z)=q_{d}(z) I$ with SISO filter $q_{d}(z) \in \mathcal{R} \mathcal{L}_{\infty}$ leads to the next result.

Corollary 1. Assume $Q(z)=q_{d}(z) I \in \mathcal{R L}_{\infty}^{n \times n}$ with SISO filter $q_{d}(z) \in \mathcal{R} \mathcal{L}_{\infty}$. The iteration (3) converges iff

$$
\left|q_{d}\left(e^{\iota \omega}\right)\right| \rho\left(I-L\left(e^{\iota \omega}\right) J\left(e^{\iota \omega}\right)\right)<1 \quad \forall \omega \in[0, \pi],
$$

and converges monotonically w.r.t. the $\ell_{2}$ norm of $f_{j}$ iff

$$
\left|q_{d}\left(e^{\iota \omega}\right)\right| \bar{\sigma}\left(I-L\left(e^{\imath \omega}\right) J\left(e^{\iota \omega}\right)\right)<1 \quad \forall \omega \in[0, \pi] .
$$

Corollary 1 enables SISO design of $q_{d}(z)$ that guarantees robust convergence of the MIMO system using $\hat{J}_{\mathrm{FRF}}\left(e^{\imath \omega}\right)$. This leads to the following design algorithm, constituting step (4).

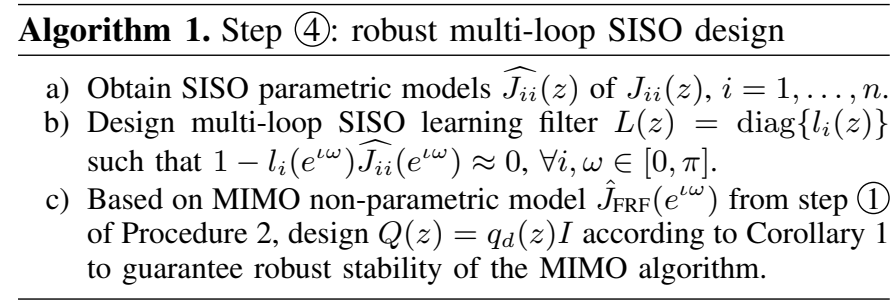

To conclude, convergence can be guaranteed. However, performance may be limited: Corollary 1 is very restrictive on the structure of $Q$. This motivates the development of decentralized designs, where each loop is robustified individually.

\section{DeCentralized ILC: Robustness to Interaction THROUGH INDEPENDENT $Q$-FILTER DESIGNS}

For systems where interaction cannot be ignored in view of convergence, yet high performance is desired using only SISO parametric models, decentralized ILCs can be designed, see (5). In this section, a decentralized design approach is developed that guarantees robust convergence of the MIMO system. The approach requires the same models as Algorithm 1: user effort (R3) is only increased by more involved computations.

In this section, the focus is on decentralized design of $Q(z) \in \mathcal{R} \mathcal{L}_{\infty}^{n_{u} \times n_{u}}$ for given $L(z) \in \mathcal{R} \mathcal{L}_{\infty}^{n_{u} \times n_{y}}$, which can be diagonal or full MIMO, see steps (5) and (6). Yet, the results are foreseen to be most often applied to square systems, since this also enables decentralized design of $L=\operatorname{diag}\left\{l_{1}, l_{2}, \ldots, l_{n}\right\}$.

\section{A. Factorization of Iteration Dynamics}

To analyze the role of interaction, (3) is factored as

$$
f_{j+1}=Q M f_{j}+\tilde{w}=Q M_{\mathrm{d}}(I+E) f_{j}+\tilde{w}
$$

where $M=I-L J, M_{\mathrm{d}}=\operatorname{diag}\left\{M_{i i}\right\}$ consists of the diagonal elements of $M, E=M_{\mathrm{d}}^{-1}\left(M-M_{\mathrm{d}}\right)$ contains the normalized interaction in $M$, and $\tilde{w}=Q L S r$, see Figure 4 . Note that $M_{\mathrm{d}}$ and $E$ are functions of $J$ and $L$, and $E=0$ if $J$ is diagonal, i.e., there is no interaction. The interaction term $I+E$ can be used to analyze robust stability. The following result is the basis for forthcoming decentralized designs.

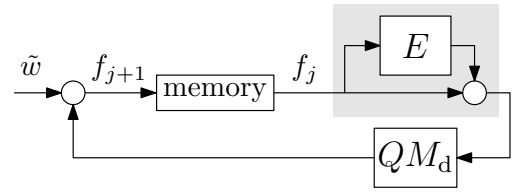

Fig. 4. Schematical representation of the factored iteration (12). Since the interaction term $I+E$ (grey area) is invariant to $Q$, measures on $I+E$ can be developed to design decentralized filters $Q$ for robust convergence.

\section{Lemma 1. Iteration (3) converges iff}

$$
\rho\left(Q M_{\mathrm{d}}(I+E)\right)<1 \quad \forall \omega \in[0, \pi] .
$$

and converges monotonically w.r.t. the $\ell_{2}$ norm of $f_{j}$ iff

$$
\rho\left(M_{\mathrm{d}}^{H} Q^{H} Q M_{\mathrm{d}}(I+E)(I+E)^{H}\right)<1 \quad \forall \omega \in[0, \pi],(14)
$$

where the superscript $H$ denotes conjugate transpose.

Proof. (13) follows directly from substituting (12) into (6), and (14) follows from substituting (12) into (8), and rewriting:

$$
\begin{aligned}
\bar{\sigma}(Q(I-L J)) & =\sqrt{\rho\left(Q M(Q M)^{H}\right)}=\sqrt{\rho\left(Q M M^{H} Q^{H}\right)} \\
= & \sqrt{\rho\left(Q M_{\mathrm{d}}(I+E)(I+E)^{H} M_{\mathrm{d}}^{H} Q^{H}\right)} \\
= & \sqrt{\rho\left(M_{\mathrm{d}}^{H} Q^{H} Q M_{\mathrm{d}}(I+E)(I+E)^{H}\right)},(15 \mathrm{a})
\end{aligned}
$$

where it is used that $\left\{\lambda_{i}(A B)\right\}=\left\{\lambda_{i}(B A)\right\}$ for square $A, B$. Substituting (15a) in (8) and squaring completes the proof.

Two observations are made for forthcoming developments:

- The matrix $Q M_{\mathrm{d}}$ in (13), respectively $M_{\mathrm{d}}^{H} Q^{H} Q M_{\mathrm{d}}$ in (14), has diagonal structure and is right multiplied with interaction term $(I+E)$, respectively $(I+E)(I+E)^{H}$.

- Comparing with (8), condition (14) is based on a spectral radius $\rho(\cdot)$ instead of a maximum singular value $\bar{\sigma}(\cdot)$.

Together, the structured form and the use of $\rho(\cdot)$ allow for the development of robust decentralized design techniques.

Remark 2. The factorization (12) resembles decentralized feedback control, see, e.g., [21] and [30, Section 10.6], yet fundamentally differs regarding the use of $E$.

In decentralized feedback design, i.e., $K=\operatorname{diag}\left\{k_{i}\right\}$ with open-loop transfer $G K$, the return difference is factored as

$$
I+G K=(I+E \tilde{T})\left(I+G_{\mathrm{d}} K\right),
$$

where $E=\left(G-G_{\mathrm{d}}\right) G_{\mathrm{d}}^{-1}, G_{\mathrm{d}}=\operatorname{diag}\left\{G_{i i}\right\}$, and $\tilde{T}=(I+$ $\left.G_{\mathrm{d}} K\right)^{-1} G_{\mathrm{d}} K$. Assuming that $\tilde{T}$ is stable, the closed-loop $T=$ $I-S$ is stable if $\rho(E \tilde{T})<1, \forall \omega \in[0, \pi]$, see [21, Theorem

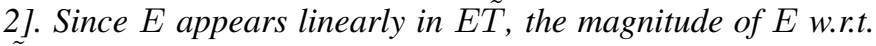
$\tilde{T}$ is typically used to analyze stability, see, e.g., [21].

In decentralized ILC, (12) is affine in E, and bounds on $I+E$ w.r.t. $Q M_{\mathrm{d}}$ are developed. The key difference is that (12) is factored, which is the open-loop in iteration domain, while in feedback the closed-loop return difference (16) is factored.

\section{B. Decentralized Conditions for Robust Convergence}

Next, several decentralized design conditions are developed. The conditions are less conservative than Corollary 1 since the decentralized structure of $Q$ is explicitly taken into account. 
1) Independent Q-filter Design Based on Induced Norms: In this subsection, upper bounds on the spectral radii in (13) and (14) based on induced norms are used for decentralized design of $Q$. In particular, for any matrix $A$, it holds $\rho(A) \leq$ $\|A\|_{i p}$. This relation is crucial for the presented designs.

Theorem 4. Iteration (3) with decentralized filter $Q(z)=$ $\operatorname{diag}\left\{q_{i}(z)\right\}$, as in (9), converges if either:

$$
\begin{array}{ll}
\left|q_{i}\left(e^{\iota \omega}\right) M_{i i}\left(e^{\iota \omega}\right)\right|<\frac{1}{\sum_{j}\left|I+E\left(e^{\iota \omega}\right)\right|_{i j}} \quad \forall i, \omega \in[0, \pi], \\
\left|q_{i}\left(e^{\iota \omega}\right) M_{i i}\left(e^{\iota \omega}\right)\right|<\frac{1}{\sum_{j}\left|I+E\left(e^{\iota \omega}\right)\right|_{j i}} \quad \forall i, \omega \in[0, \pi],
\end{array}
$$

and converges monotonically w.r.t. the $\ell_{2}$ norm of $f_{j}$ if

$$
\begin{array}{r}
\left|q_{i}\left(e^{\iota \omega}\right) M_{i i}\left(e^{\iota \omega}\right)\right|<\frac{1}{\sqrt{\sum_{j}\left|\left(I+E\left(e^{\iota \omega}\right)\right)\left(I+E\left(e^{\iota \omega}\right)\right)^{H}\right|_{i j}}} \\
\forall i, \omega \in[0, \pi],
\end{array}
$$

where $|\cdot|$ denotes element-wise absolute value.

Proof. Conditions (17), (18) follow by application of $\rho(A) \leq$ $\|A\|_{i p}$ to (13) with $p=\infty, p=1$, respectively. Similarly, (19) follows from (14), for both $p=1, p=\infty$.

Remark 3. Theorem 4 presents the ILC-analog of Gershgorin bounds in feedback control, see, e.g., [21], [30, Section 10.6].

2) Independent $Q$-filter Design Based on the SSV: Alternatively, conditions are developed using the structured singular value (SSV), see, e.g., [21], [22]. The idea is to exploit the diagonal structure of $Q M_{\mathrm{d}}$ in Lemma 1. Particularly, for a matrix $A$ and diagonal matrix $\Delta$, see [30, eq. (8.95)], it holds

$$
\rho(\Delta A) \leq \bar{\sigma}(\Delta) \mu_{\Delta}(A)
$$

where $\mu_{\Delta}(A)$ is taken with respect to the structure of $\Delta$.

Definition 3. For $A \in \mathbb{C}^{n \times n}$, the $S S V \mu_{\Delta}(A)$ is defined

$$
\mu_{\Delta}(A)=\frac{1}{\min \{\bar{\sigma}(\Delta): \Delta \in \Delta, \operatorname{det}(I-A \Delta)=0\}},
$$

where $\Delta$ is a prescribed set of block diagonal matrices, unless no $\Delta \in \Delta$ makes $I-A \Delta$ singular, in which case $\mu_{\Delta}(A)=0$.

Theorem 5. Iteration (3) with decentralized filter $Q(z)=$ $\operatorname{diag}\left\{q_{i}(z)\right\}$, as in (9), converges if

$$
\left|q_{i}\left(e^{\iota \omega}\right) M_{i i}\left(e^{\iota \omega}\right)\right|<\frac{1}{\mu_{\mathrm{d}}\left(I+E\left(e^{\iota \omega}\right)\right)} \quad \forall i, \omega \in[0, \pi],
$$

and converges monotonically w.r.t. the $\ell_{2}$ norm of $f_{j}$ if

$$
\begin{gathered}
\left|q_{i}\left(e^{\iota \omega}\right) M_{i i}\left(e^{\iota \omega}\right)\right|<\frac{1}{\sqrt{\mu_{\mathrm{d}}\left(\left(I+E\left(e^{\iota \omega}\right)\right)\left(I+E\left(e^{\iota \omega}\right)\right)^{H}\right)}} \\
\forall i, \omega \in[0, \pi],
\end{gathered}
$$

where $\mu_{\mathrm{d}}(\cdot)$ is the SSV with respect to a diagonal structure.

Proof. Using (20) in (13), where $\Delta=Q M_{\mathrm{d}}, A=I+E$ and $\boldsymbol{\Delta}=\left\{\delta I: \delta \in \mathbb{C}^{n}\right\}$, gives

$$
\rho\left(Q\left(e^{\iota \omega}\right) M_{\mathrm{d}}\left(e^{\iota \omega}\right)\right)<\frac{1}{\mu_{\mathrm{d}}\left(I+E\left(e^{\iota \omega}\right)\right)} \quad \forall \omega \in[0, \pi] .
$$

Omitting arguments, $\rho\left(Q M_{\mathrm{d}}\right)=\max _{i}\left|q_{i} M_{i i}\right|$ implies (21). Applying (20) to (14), observing that $\bar{\sigma}\left(M_{\mathrm{d}}^{H} Q^{H} Q M_{\mathrm{d}}\right)=$ $\max _{i}\left|q_{i} M_{i i}\right|^{2}$, and taking square roots proves (22).

The SSV is employed in a fundamentally different way than in stability analyses of feedback systems. In robust control, e.g., [22, Chapters 9, 11], typically $\mu_{\Delta}(M)$ is taken with respect to structured uncertainty $\Delta$, and $M$ denotes a nominal model. In contrast, here $I+E$ has the role of nominal model, and $Q M_{\mathrm{d}}$ is the structured uncertainty yet to be designed.

\section{Decentralized Q-filter Design for Robustness to Interaction}

Theorems 4 and 5 enable systematic and robust (R1) decentralized design, using only SISO parametric models (R3). This is summarized as follows, constituting step (5) of Procedure 2.

\begin{tabular}{l}
\hline Algorithm 2. Step (5): robust decentralized MIMO design \\
\hline a) Obtain SISO parametric models $\widehat{J_{i i}}(z)$ of $J_{i i}(z), i=1, \ldots, n$. \\
b) Design $L(z)=\operatorname{diag}\left\{l_{i}(z)\right\}$ such that $1-l_{i} \widehat{J_{i i}} \approx 0, \forall i$. \\
c) Construct $M_{\mathrm{d}}\left(e^{\iota \omega}\right)$ and $E\left(e^{\iota \omega}\right)$, see $(12)$, based on $L$ from b) \\
and MIMO FRF model $\hat{J}_{\mathrm{FRF}}\left(e^{\iota \omega}\right)$ from step $(1$ of Procedure 2. \\
d) For robust stability, design $Q(z)=\operatorname{diag}\left\{q_{i}\right\}$ according to joint \\
evaluation of Theorems 4,5, i.e., for each frequency $\omega \in[0, \pi]$, \\
- at least one of (17), (18), (21) is satisfied (convergence); \\
- at least one of (19), (22) is satisfied (monotonic convergence).
\end{tabular}

The key advantage of Algorithm 2, compared to Algorithm 1 , is that performance (R2) can potentially be increased, while the modeling requirements (R3) remain equal. Indeed, (17)-(19) and (21)-(22) can be computed using $\hat{J}_{\mathrm{FRF}}\left(e^{\iota \omega}\right)$, such that interaction does not have to be included in models $\widehat{J_{i i}}(z)$.

Remark 4. In Algorithm 2, the developed bounds (17), (18), (21), respectively (19), (22), are jointly considered. This is since the ordering of their tightness may vary as a function of frequency, and hence they all contribute to the design. Note however that, for a specific frequency $\omega \in[0, \pi]$, they can in general not be combined over the different SISO loops $i$. That is, convergence is guaranteed only if, per evaluated frequency, at least one condition is satisfied for all loops i simultaneously.

Remark 5. In the SISO case, the results in Theorems 4 and 5 recover the SISO condition (10), since in this case $E=0$.

The achievable performance of decentralized ILC, i.e., the magnitude of $e_{\infty}$, is limited by interaction that is ignored in the design of $L$. If increased modeling effort is justified (R3) in relation to performance requirements (R2), MIMO parametric models of $J$ can be used to design centralized ILC algorithms.

\section{Centralized ILC: AcCounting For Interaction THROUGH $L$-FILTER DESIGNS}

For systems where decentralized ILC yields unsatisfactory performance due to required robustness to ignored interaction, and increased modeling effort (R3) is justified in view of performance requirements (R2), centralized ILC schemes can be designed using a full MIMO model, i.e., step (6). By explicitly accounting for interaction in $L$, the requirement for robustness through $Q$ is alleviated, which potentially increases performance. This leads to the following algorithm.

\begin{tabular}{l}
\hline Algorithm 3. Step (6): centralized MIMO design \\
\hline a) Obtain MIMO parametric model $\hat{J}(z)$, including interaction; \\
b) Design $L(z)$ such that $I-L \hat{J} \approx 0$, e.g., [17], [23], [24]. \\
c) For robust stability, design $Q(z)$ according to Corollary 1, \\
Theorem 4 or 5 based on FRF model $\hat{J}_{\mathrm{FRF}}\left(e^{\iota \omega}\right)$ from step (1).
\end{tabular}

In preceding sections, the techniques underlying steps (4) to (6) are developed. Next, these are applied to the case study. 


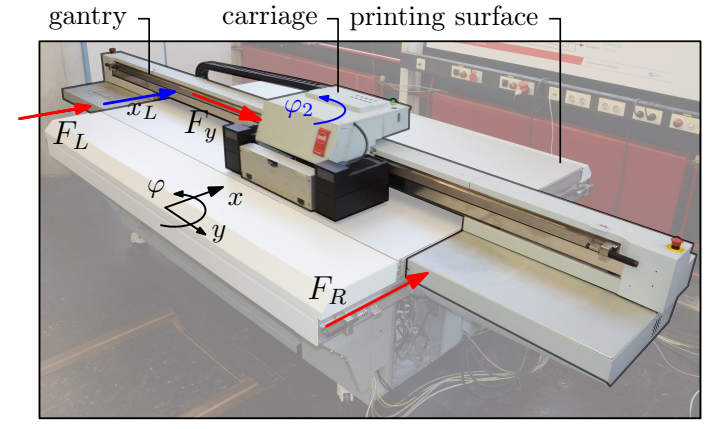

Fig. 5. Océ Arizona 550GT flatbed printer. The carriage moves along the gantry, which provides the motion freedom to cover the printing surface. The actuator forces are indicated by red arrows. The inputs considered for control are $F_{L}, F_{R}$, and the outputs are $x_{L}, \varphi_{2}$, indicated by blue arrows.

\section{ViI. Application of Design Framework to Multivariable Case Study}

In this section, Procedure 2 is applied to a case study in a step-by-step manner. Simulations are performed to clearly show the differences between the developed approaches. Details on the case study and Matlab implementations of Procedure 2 are available as Supplementary Material.

\section{A. Case Study: Océ Arizona 550GT Flatbed Printer}

An Océ Arizona 550GT printer is considered, see Figure 5. In contrast to standard consumer printers, the printer can print on both flexible and rigid media, e.g., paper, plastics, wood and metals. The medium is fixed on the printing surface, and the carriage, which contains the printheads, moves in the horizontal plane. This yields inherently multivariable dynamics.

The simulations are performed using the model shown in Figure 6. The inputs are forces $F_{L}[\mathrm{~N}]$ and $F_{R}[\mathrm{~N}]$ acting on the gantry; the outputs are the gantry position at the left side $x_{L}[\mathrm{~m}]$, and carriage rotation $\varphi_{2}[\mathrm{rad}]$, i.e., $\left[\begin{array}{l}x_{L} \\ \varphi_{2}\end{array}\right]=G_{o}\left[\begin{array}{l}F_{L} \\ F_{R}\end{array}\right]$, where $G_{o}$ is the system before decoupling in step (3).

The system is discretized using zero-order-hold on the input with sampling interval $10^{-3} \mathrm{~s}$. A stabilizing diagonal feedback controller $C(z)=\operatorname{diag}\left\{c_{1}(z), c_{2}(z)\right\}$ is designed, where

$c_{1}(z)=\frac{5 \times 10^{4}(z-0.988)}{z-0.939}, c_{2}(z)=\frac{1.3 \times 10^{4}(z-0.991)}{z-0.969}$,

yielding a bandwidth of $3 \mathrm{~Hz}$ in $x_{L}$ direction and $1.5 \mathrm{~Hz}$ in $\varphi_{2}$ direction. The system $J(z)=(I+G C)^{-1} G$ has nonminimum phase transmission zeros at $z=1.09$ and $z=-6.69$ due to the non-collocated inputs/outputs and fast sampling. A model $\hat{J}(z)=(I+\hat{G} C)^{-1} \hat{G}$ is provided for ILC design, see Figure 6. A modeling error is present at the first resonance in the $(2,2)$-element, which plays a crucial role in the designs.

\section{B. Results: Application of Procedure 2 to Case Study}

Next, Procedure 2 is step-by-step applied to the case study, and the results are presented. The disturbance $r=\left[r_{x}, r_{\varphi}\right]^{\top}$ of length $N=3001$ is shown in Figure 7. An overview of the designs is provided in Table I, and the resulting performance $\left\|e_{j}\right\|_{F}$ is shown in Figure 3, where $\left\|e_{j}\right\|_{F}=\sqrt{\sum_{i, k}\left|e_{j}(i, k)\right|^{2}}$

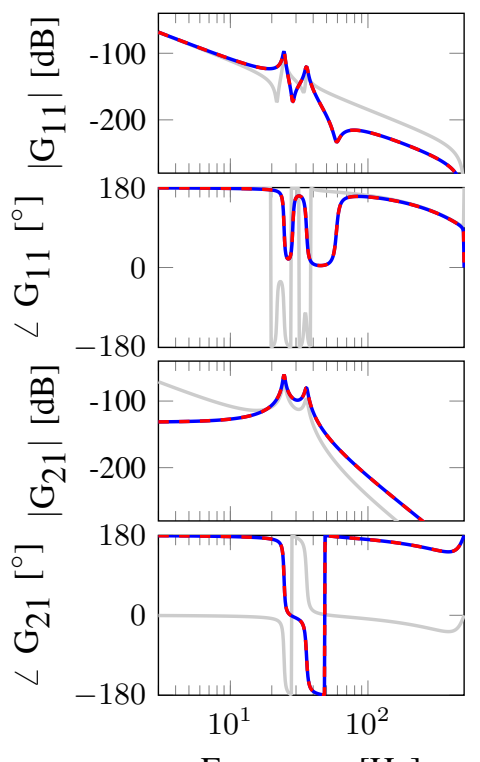

Frequency $[\mathrm{Hz}]$

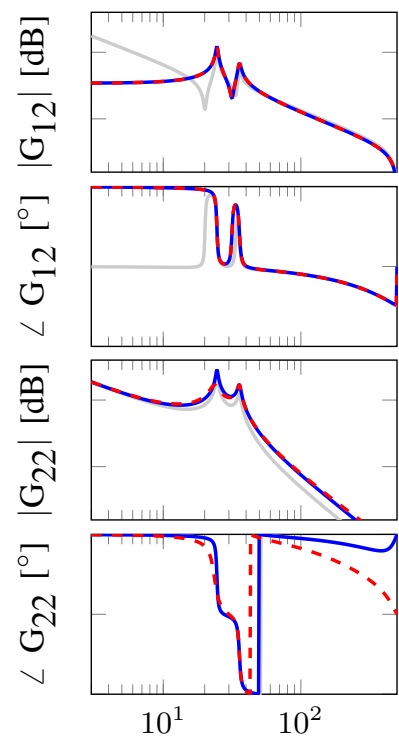

Frequency $[\mathrm{Hz}]$
Fig. 6. Bode diagram of non-decoupled true plant $G_{O}(z)(-)$, true plant $G$ (-) after decoupling transformations in step (3) of Procedure 2, and model of decoupled plant $\widehat{G}(z)(--)$ used for ILC design.

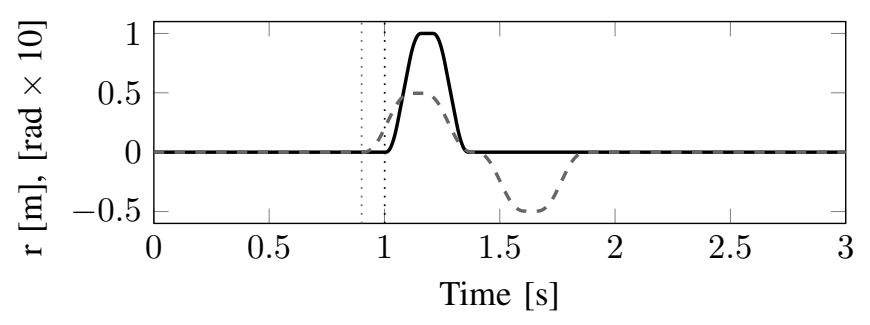

Fig. 7. Reference trajectories $r_{x}(-)$ and $r_{\varphi}(--)$. The start of the motion tasks are indicated by dotted lines.

with $e_{j}=\left[e_{j, x}, e_{j, \varphi}\right]^{\top} \in \mathbb{R}^{N \times 2}$. Note that $e_{x}[\mathrm{~m}]$ and $e_{\varphi}[\mathrm{rad}]$ are weighed equally since they have comparable magnitude.

(1) Non-parametric modeling: it is assumed that the MIMO non-parametric FRF model is exact, i.e., $\hat{J}_{\mathrm{FRF}}\left(e^{\iota \omega}\right)=J\left(e^{\iota \omega}\right)$.

(2) Interaction analysis. From Figure 6, it can be directly observed that there is substantial interaction above $20 \mathrm{~Hz}$.

(3) Decoupling transformations. The input is transformed using static matrix $T_{u}$, see, e.g., [20], such that plant $G=G_{o} T_{u}$ is diagonally dominant at low frequencies, see Figure 6.

(4) Robust multi-loop SISO design. The filters $l_{i}=1 / \hat{J}_{i i}$ are implemented using stable inversion, see, e.g., [17]. Filters $q_{i}$ are first-order zero-phase low-pass Butterworth filters.

- Independent SISO schemes $(X,-)$ are designed according to (10) and Procedure 1, see Figure 10(a). Since interaction is ignored, convergence is not guaranteed (R1), see Figure 10(b). This is corroborated by Figure 3.

- Through robust SISO design ( $\square,-)$ according to Algorithm 1, convergence is guaranteed using MIMO FRF model $\hat{J}_{\mathrm{FRF}}\left(e^{\iota \omega}\right)$, see Figure 10(b). Note that $q_{d}$ is cut off at a low frequency due to required robustness in loop 2. This comes at the cost of performance (R2), also in loop 1, see Figure 8 . 
TABLE I

OVERVIEW OF APPROACHES IN DESIGN FRAMEWORK: MODELING REQUIREMENTS, DESIGN PARAMETERS, AND ASYMPTOTIC PERFORMANCE.

\begin{tabular}{|c|c|c|c|c|c|c|c|c|c|c|}
\hline & \multirow[b]{2}{*}{$L$-filter } & \multirow{2}{*}{$\begin{array}{l}\text { required } \\
\text { parametric } \\
\text { models }\end{array}$} & \multirow[b]{2}{*}{$Q$-filter } & \multirow{2}{*}{$\begin{array}{c}\text { guaranteed } \\
\text { robust } \\
\text { stability (R1) }\end{array}$} & \multirow{2}{*}{$\begin{array}{l}\text { performance } \\
\text { (R2) }\end{array}$} & \multirow{2}{*}{$\begin{array}{l}\text { user effort } \\
\text { (R3) }\end{array}$} & \multicolumn{2}{|c|}{ cut-off $f_{c}[\mathrm{~Hz}]$} & \multirow[b]{2}{*}{$\left\|e_{\infty}\right\|_{F}$} & \\
\hline & & & & & & & $q_{1}$ & $q_{2}$ & & \\
\hline Proc. 1 & $n \times$ SISO & $n \times \mathrm{SISO}$ & SISO, $\S$ IV-A & no & - & + & 100 & 15 & N/A & $x$ \\
\hline Proc. 2: (4) & $n \times$ SISO & $n \times$ SISO & Robust SISO, §IV-B & yes & - & + & 13 & 13 & 0.45 & $\square$ \\
\hline Proc. 2: (5) & $n \times$ SISO & $n \times$ SISO & Decentralized, $\S \mathrm{V}$ & yes & $+1-$ & $+/-$ & 19 & 9 & 0.30 & 0 \\
\hline Proc. 2: (6) & MIMO, $\S \mathrm{VI}$ & full MIMO & Robust SISO, $\S I V-B$ & yes & + & - & 28 & 28 & 0.14 & $\diamond$ \\
\hline
\end{tabular}

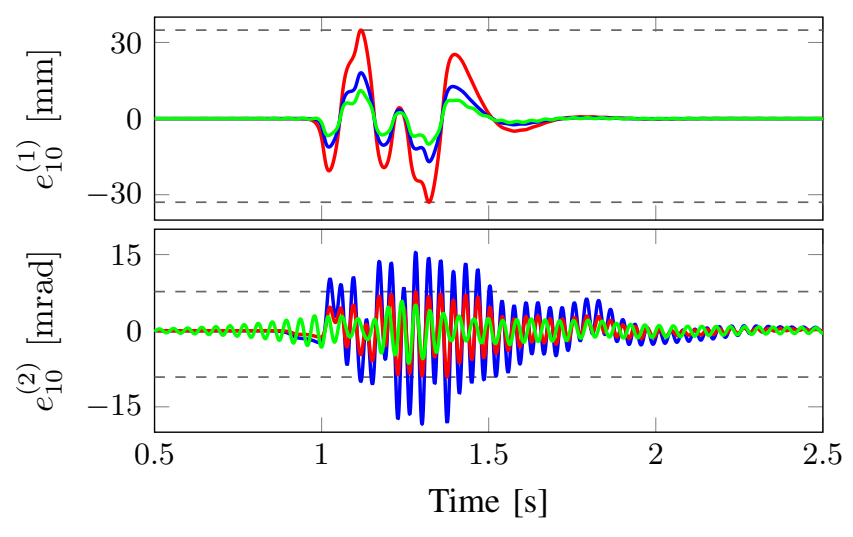

Fig. 8. Error signals in trial $j=10$ of robust SISO design in step (4) (-), decentralized design in step (5) (-) and centralized design in step (6) (-).

(5) Decentralized robust MIMO design $(\mathrm{O},-$ ) using Algorithm 2 further improves performance, see Figure 3. The same models are used: only decentralized filter $Q(z)=\operatorname{diag}\left\{q_{i}(z)\right\}$ is designed in a more sophisticated manner, see Figure 10(c).

- Compared to robust SISO design in (4), the cut-off frequency of $q_{1}$ is significantly higher, see Table I. In loop 2, the modeling error is dominant beyond $10 \mathrm{~Hz}$, whereas in loop 1 robustness is required to interaction above $20 \mathrm{~Hz}$.

- The main improvement is achieved in loop 1, see Figure 8, whereas the error in loop 2 is slightly increased.

- In view of d) of Algorithm 2, note that each condition (17), (18), (21), is violated at least once over the frequency range, see Figure 10(c). Hence, they all contribute to the design.

(6) Centralized MIMO design $(\diamond,-)$ using Algorithm 3 yields the highest performance. Given MIMO model $\hat{J}$, the MIMO filter $L=\hat{J}^{-1}$ is implemented using stable inversion.

- By designing for interaction in $L$, less robustness is required compared to (4) and (5): the cut-off frequency of $Q=q_{d} I$, see Corollary 1, is significantly higher, see Figure 10(d).

- The required MIMO model $\hat{J}$ can be expensive to obtain in practice: user effort (R3) is sacrificed for performance (R2).

- All approaches generate pre-actuation to compensate the non-minimum phase transmission zeros of $J$, see Figure 9.

The following key conclusions are made: i) interaction must be taken into account in the design, ii) performance can be improved with limited user effort through decentralized designs, and iii) if justified by performance requirements, performance can be further improved through centralized MIMO design.

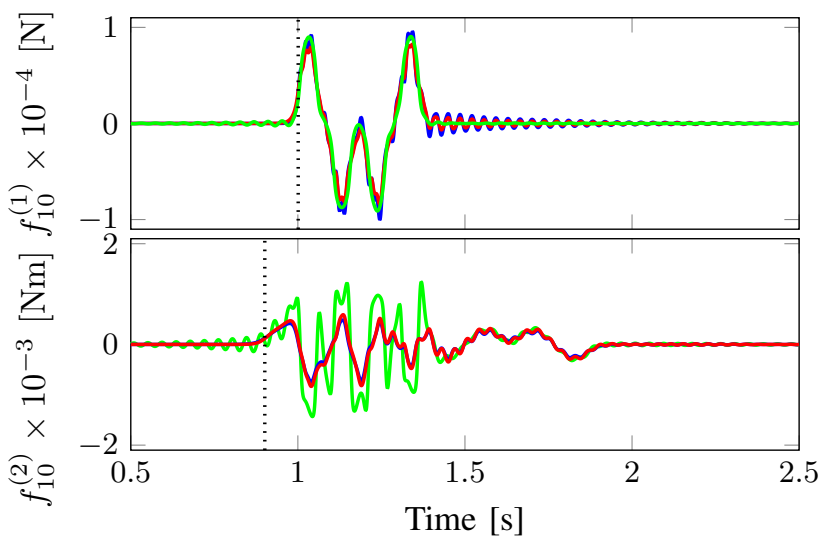

Fig. 9. Feedforward signals in trial $j=10$ of the robust SISO design in step (4) (-), decentralized design in step (5) (-) and centralized design in step (6) (-). The start of the motion tasks are indicated by dotted lines.

\section{CONCLUSIONS}

The design framework developed in this paper enables systematic design of ILC controllers for multivariable systems, and balances performance requirements with modeling and design effort through a range of design solutions. This is done by judiciously combining non-parametric FRF measurements and parametric models. The results are demonstrated on a flatbed printing system, including trade-offs between approaches.

\section{ACKNOWLEDGMENT}

The authors thank Sjirk Koekebakker, Maarten Steinbuch, Jeroen Willems and Jurgen van Zundert for their contributions.

\section{REFERENCES}

[1] K. Barton, D. Hoelzle, A. Alleyne, and A. Johnson, "Cross-coupled iterative learning control of systems with dissimilar dynamics: design and implementation," Int. J. Control, vol. 84, no. 7, pp. 1223-1233, 2011.

[2] G. M. Clayton, S. Tien, K. Leang, Q. Zou, and S. Devasia, "A review of feedforward control approaches in nanopositioning for high-speed SPM,” J. Dyn. Syst. Meas. Control, vol. 131, no. 6, pp. 061 101-1061 101-19, 2009.

[3] J. van Zundert, J. Bolder, S. Koekebakker, and T. Oomen, "Resourceefficient ILC for LTI/LTV systems through LQ tracking and stable inversion: Enabling large feedforward tasks on a position-dependent printer," Mechatronics, vol. 38, pp. 76 - 90, 2016.

[4] D. de Roover and O. Bosgra, "Synthesis of robust multivariable iterative learning controllers with application to a wafer stage motion system," Int. J. Control, vol. 73, no. 10, pp. 968-979, 2000.

[5] K. Moore, Iterative Learning Control for Deterministic Systems. Springer, 1993. 


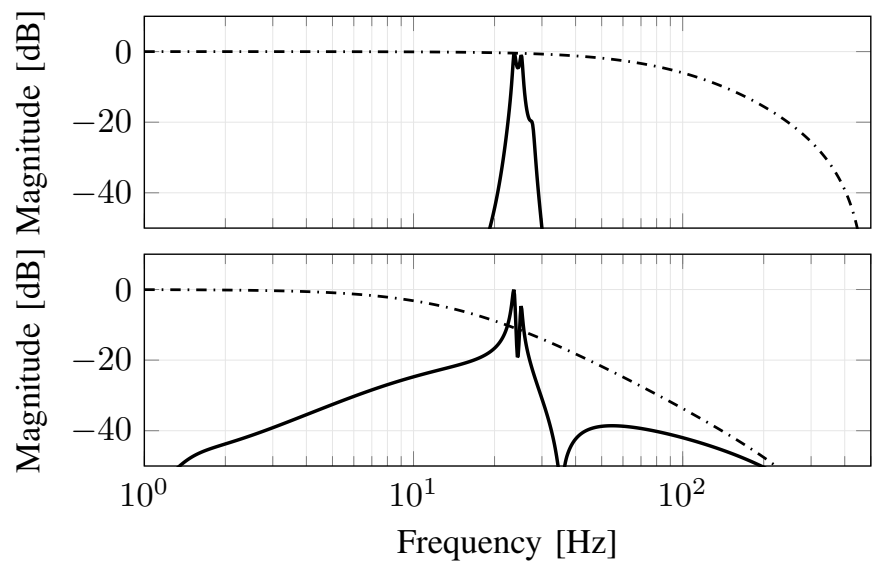

(a) Interaction-ignoring SISO design using (10): filters $q_{i}(-\cdot-)$ (top: $i=1$; bottom: $i=2)$ are designed such that $\left|q_{i}\left(1-l_{i} \hat{J}_{\mathrm{FRF}, \mathrm{ii}}\right)\right|<1, \forall \omega(-)$.

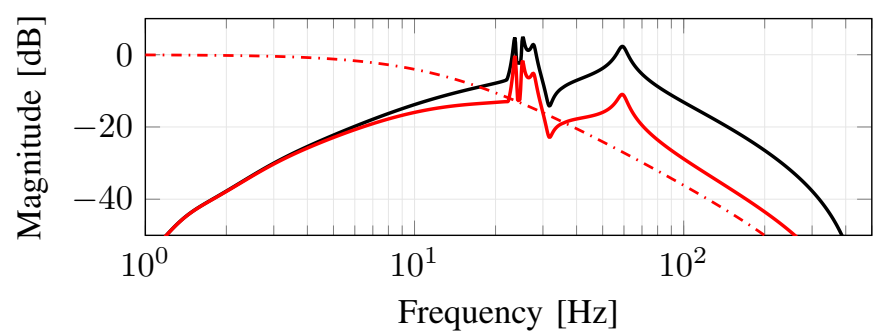

(b) Step (4): robust SISO design of $Q=q_{d} I$ (---) according to Algorithm 1 guarantees convergence, i.e., $\rho\left(Q\left(I-L J_{\mathrm{FRF}}\right)\right)<1, \forall \omega(-)$, in contrast to interaction-ignoring SISO designs (-), see Figure 10(a).

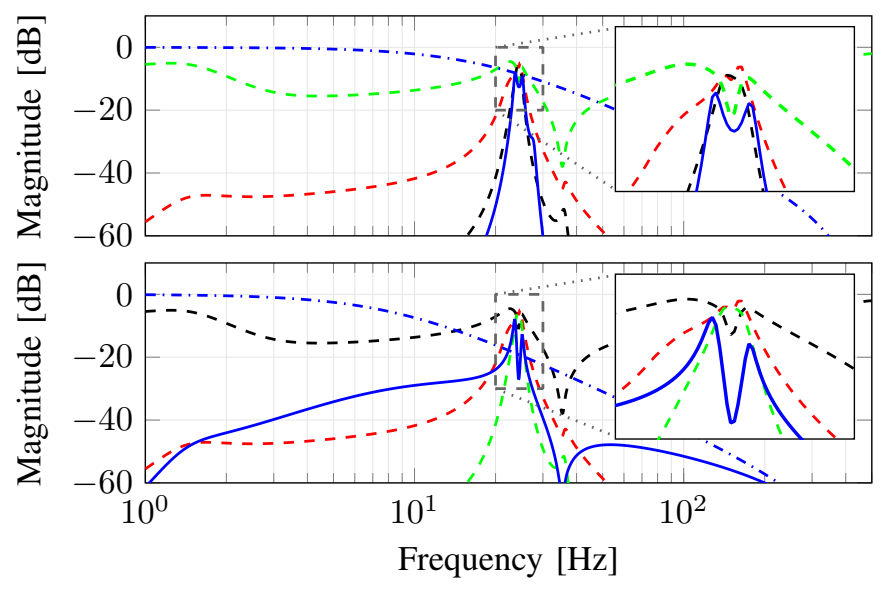

(c) Step (5): robust decentralized design according to Algorithm 2: the filters $q_{i}(--)$ (top: $i=1$; bottom: $i=2$ ) are chosen such that, for each frequency, $\left|q_{i}\left(1-l_{i} \hat{J}_{\mathrm{FRF}, \mathrm{ii}}\right)\right|=\left|q_{i} M_{i i}\right|(-)$ are upper bounded by at least one of the right-hand sides of $(17),(18)$ and $(21)(--,--,--$, respectively).

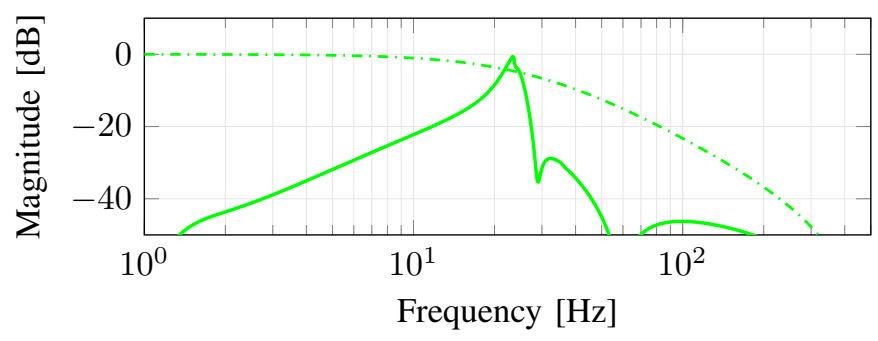

(d) Step (6): centralized ILC design according to Algorithm 3 and (11): the filter $Q=q_{d} I(-\cdot)$ is designed such that $\left|q_{d}\right| \rho\left(1-L \hat{J}_{\mathrm{FRF}}\right)<1, \forall \omega(-)$.
[6] D. H. Owens, C. T. Freeman, and B. Chu, "Multivariable norm optimal iterative learning control with auxiliary optimisation," Int. J. Control, vol. 86, no. 6, pp. 1026-1045, 2013.

[7] X. Li, D. Huang, B. Chu, and J.-X. Xu, "Robust iterative learning control for systems with norm-bounded uncertainties," Int. J. Robust Nonlin., vol. 26, no. 4, pp. 697-718, 2016.

[8] T. Son, G. Pipeleers, and J. Swevers, "Robust monotonic convergent iterative learning control," IEEE Trans. Automat. Contr., vol. 61, no. 4, pp. 1063-1068, 2016.

[9] D. H. Owens, Iterative Learning Control: An Optimization Paradigm, ser. Advances in Industrial Control. Springer-Verlag London, 2016.

[10] P. Janssens, G. Pipeleers, and J. Swevers, "A data-driven constrained norm-optimal iterative learning control framework for LTI systems," IEEE Trans. Contr. Syst. Technol., vol. 21, no. 2, pp. 546-551, 2013.

[11] H. Hjalmarsson, "From experiment design to closed-loop control," Automatica, vol. 41, no. 3, pp. 393 - 438, 2005.

[12] J. Bolder, S. Kleinendorst, and T. Oomen, "Data-driven multivariable ILC: enhanced performance by eliminating L and Q filters," Int. J. Robust Nonlin., vol. 28, no. 12, pp. 3728-3751, 2018.

[13] R. Pintelon and J. Schoukens, System identification: a frequency domain approach. John Wiley \& Sons, 2012.

[14] N. Strijbosch, L. Blanken, and T. Oomen, "Frequency domain design of iterative learning control and repetitive control for complex motion systems," in IEEJ International Workshop on Sensing, Actuation, Motion Control, and Optimization (SAMCON), Tokyo, Japan, 2018.

[15] J. Wallén, M. Norrlof, and S. Gunnarsson, "Arm-side evaluation of ILC applied to a six-degrees-of-freedom industrial robot," in Proceedings of the 17th IFAC World Congress, Seoul, Korea, 2008, pp. 13450-13455.

[16] W. Paszke, E. Rogers, K. Gałkowski, and Z. Cai, "Robust finite frequency range iterative learning control design and experimental verification," Contr. Eng. Prac., vol. 21, no. 10, pp. 1310 - 1320, 2013.

[17] J. van Zundert and T. Oomen, "On inversion-based approaches for feedforward and ILC," IFAC Mechatronics, vol. 50, pp. 282-291, 2018.

[18] Y. Fang and T. W. Chow, "Iterative learning control of linear discretetime multivariable systems," Automatica, vol. 34, no. 11, pp. 1459 1462, 1998.

[19] T. V. Dinh, C. T. Freeman, and P. L. Lewin, "Assessment of gradientbased iterative learning controllers using a multivariable test facility with varying interaction," Contr. Eng. Prac., vol. 29, pp. 158 - 173, 2014.

[20] T. Oomen, "Advanced motion control for precision mechatronics: Control, identification, and learning of complex system," IEEJ Journal of Industry Applications, vol. 7, no. 2, 2018.

[21] P. Grosdidier and M. Morari, "Interaction measures for systems under decentralized control," Automatica, vol. 22, no. 3, pp. 309 - 319, 1986

[22] K. Zhou, J. Doyle, and K. Glover, Robust and Optimal Control. Prentice Hall, NJ, 1996.

[23] L. Blanken, S. Koekebakker, and T. Oomen, "Design and modeling aspects in multivariable iterative learning control," in Proceedings of the IEEE 55th Conference on Decision and Control, Las Vegas, NV, USA, 2016, pp. 5502-5507.

[24] L. Blanken, J. Willems, S. Koekebakker, and T. Oomen, "Design techniques for multivariable ILC: Application to an industrial flatbed printer," IFAC-PapersOnLine, vol. 49, no. 21, pp. 213 - 221, 2016, 7th IFAC Symposium on Mechatronic Systems, Loughborough, UK.

[25] S. Gunnarsson and M. Norrlöf, "On the disturbance properties of high order iterative learning control algorithms," Automatica, vol. 42, no. 11, pp. $2031-2034,2006$.

[26] T. Oomen and C. R. Rojas, "Sparse iterative learning control with application to a wafer stage: Achieving performance, resource efficiency, and task flexibility," Mechatronics, vol. 47, pp. 134 - 147, 2017.

[27] M. Norrlöf and S. Gunnarsson, "Time and frequency domain convergence properties in iterative learning control," Int. J. Control, vol. 75, no. 14 , pp. $1114-1126,2002$.

[28] J. van Zundert, F. Luijten, and T. Oomen, "Achieving perfect causal feedforward control in presence of nonminimum-phase behavior exploiting additional actuators and squaring down," in 2018 IEEE American Control Conference, Milwaukee, WI, 2018, pp. 6031-6036.

[29] J. Butterworth, L. Pao, and D. Abramovitch, "Analysis and comparison of three discrete-time feedforward model-inverse control techniques for nonminimum-phase systems," Mechatronics, vol. 22, no. 5, pp. 577-587, 2012.

[30] S. Skogestad and I. Postlethwaite, Multivariable feedback control: analysis and design, 2nd ed. Wiley, New York, 2007.

Fig. 10. Application of Procedure 2: designs of robustness filters $Q(z)$. 